\title{
The Exeter ${ }^{\mathrm{TM}}$ Universal Hip in Patients 50 Years or Younger at 10-17 Years' Followup
}

\author{
Simon C. Lewthwaite FRCS(Orth), Ben Squires FRCS(Orth), \\ Graham A. Gie FRCSEd(Orth), Andrew J. Timperley D. Phil, FRCS, \\ Robin S. M. Ling OBE, MA, BM(Oxon), FRCS
}

(C) The Association of Bone and Joint Surgeons 2008

\begin{abstract}
The Exeter ${ }^{\mathrm{TM}}$ Universal hip (Stryker Inc., Newbury, UK) has reported survival rates of $91.74 \%$ at 12 years in all patients with reoperation as an endpoint. However, its performance in younger patients has not been fully established. We reviewed survivorship and the clinical and radiographic outcomes of this hip system implanted in 107 patients (130 hips) 50 years old or younger at the time of surgery. The mean age at surgery was 42 years. The minimum followup was 10 years (mean, 12.5 years; range, 10-17 years) with no patients lost to followup. Twelve hips had been revised. Of these, nine had aseptic loosening of the acetabular component and one cup was revised for focal lysis and pain. One hip was revised for recurrent dislocation and one joint underwent revision for infection. Radiographs demonstrated 14 (12.8\%) of the remaining acetabular prostheses were loose but no femoral components were loose. Survivorship of both stem and cup
\end{abstract}

One or more of the authors (GAG, AJT, RSML) have received funding from Stryker Inc. In addition, benefits have been or will be directed to a research fund, foundation, educational institution, or other nonprofit organization with which one or more of the authors (GAG, AJT, RSML) are associated.

Each author certifies that his or her institution has approved or waived approval for the human protocol for this investigation and that all investigations were conducted in conformity with ethical principles of research.

S. C. Lewthwaite, G. A. Gie, A. J. Timperley, R. S. M. Ling

Princess Elizabeth Orthopaedic Centre, Exeter, UK

B. Squires

Taunton and Somerset Hospital, Taunton, UK

S. C. Lewthwaite $(\square)$

6 Mainwaring Close, Stapeley, Nantwich, Cheshire CW57GT, UK

e-mail: slewthy@hotmail.com from all causes was $92.6 \%$ at an average of 12.5 years. Survivorship of the stem from all causes was $99 \%$ and no stem was revised for aseptic loosening. The Exeter ${ }^{\mathrm{TM}}$ Universal stem performed well, even in the young, highdemand patient.

Level of Evidence: Level IV, therapeutic study. See the Guidelines for Authors for a complete description of levels of evidence.

\section{Introduction}

Many previous studies have demonstrated inferior survival and clinical performance of other cemented THAs when used in patients younger than 50 years $[2,6,7,13,17,20$, $40,47]$. One of the reported reasons for stem failure is the quality of cementation [3]. First-generation cementation with finger packing as described by Charnley demonstrated radiographic stem loosening of up to $24 \%$ at 5 years and $40 \%$ at 10 years [4, 48], whilst improved cementation techniques have substantially lowered this to around $3 \%$ at 11 years [38]. However, younger patients are presumed to place more demands on their implant than older patients due to higher activity levels [46] and resultant higher bearing wear rates, thus raising a question of longer-term survival beyond 10 years owing to loosening from particulate debris rather than the mechanical effects of cement technique [33].

The survivorship of the Exeter ${ }^{\mathrm{TM}}$ Universal series (Stryker Inc., Newbury, UK) femoral stem has been reported as $100 \%$ at 12.5 years [50], but its use in the younger, more active patient has never been reported fully. The Exeter stem was introduced in 1988 to offer head/neck modularity. Stem subsidence within the cement is a feature of the normal behavior of this stem and is dependent on 
loading of the joint [23]. Its geometry is similar to the original stem with no collar and a double taper extending proximally to the neck of the prosthesis, with a highly polished surface $(\mathrm{Ra}=0.01-0.03 \mu \mathrm{m})$. In 1988 , modifications were made to the shoulder, with the superolateral straight edge changed to include a curved surface, convex laterally. Beginning in 1983, the implant was made of the stronger Rex 734 [42] Orthinox ${ }^{\circledR}$ alloy (Howmedica, Rutherford, NJ), whereas the original stem was made from EN58J [22], a weaker and more ductile alloy. The stem centralizer is constructed from PMMA, is placed on the stem tip, extending 5 to $10 \mathrm{~mm}$ distally, and contains a void to allow taper engagement. Since quality of cementation is a reported predictor of outcome, we suspect that whilst it should in general be more important for a younger patient group, the load transmission of this particular stem (with anticipated subsidence and taper engagement) may be protective.

We hypothesized that Exeter ${ }^{\mathrm{TM}}$ Universal hips in young, active patients have comparable results to those used in patients of all ages for this duration of followup. Furthermore, we questioned if the implants behaved differently in younger patients as could be evidenced by greater stem subsidence, higher bearing wear rates and evidence of radiographic failure. Finally, we examined whether these higher demand patients had satisfactory clinical outcomes.

\section{Materials and Methods}

We retrospectively reviewed the medical records and radiographs of 107 selected patients (130 hips) aged 50 years and younger at the time of surgery and who had an Exeter ${ }^{\mathrm{TM}}$ Universal stem inserted by Exeter-based surgeons from 1988 to 1995 . We excluded 44 patients operated in our hospital but who lived outside the area and were operated on by non-Exeter-based surgeons since these patients did not fall within our followup control. However, no patients operated on by Exeter-based surgeons were excluded. Six patients with seven THAs died during the study period from causes unrelated to their hip surgery (210 years); their hips were functioning well both clinically and radiographically at the time of death and none had been revised. This left 101 patients (123 hips) for review. The minimum followup was 10 years (mean, 12.5 years; range, 10-17 years) with no patients lost to followup.

Patients had a variety of diagnoses and all were included for evaluation (Table 1). Seventy-seven percent had a primary diagnosis other than osteoarthritis. Previous surgery had been performed in $32 \%$ of cases and included arthrodesis, femoral and acetabular osteotomy, and débridement for septic arthritis. Because of the prevalence of abnormal anatomy, $15 \%$ of hips underwent major
Table 1. Primary diagnosis

\begin{tabular}{lcc}
\hline Diagnosis & Number & Percent \\
\hline Primary osteoarthritis & 43 & 33.1 \\
Dysplasia & 40 & 30.7 \\
Inflammatory arthritis & 24 & 18.5 \\
Legg-Calvé-Perthes & 6 & 4.6 \\
Avascular necrosis & 4 & 3.1 \\
Slipped upper femoral epiphysis & 3 & 2.3 \\
Septic arthritis & 3 & 2.3 \\
Other & 7 & 5.4 \\
\hline
\end{tabular}

acetabular reconstruction with structural grafts and fixation (Fig. 1A-B). Thirty-two percent of the patients were in Charnley Group C [11] with their function affected by systemic disease, $25 \%$ were in Charnley Group B with both hips involved, and 32\% were in Charnley Group A with one hip involved.

All surgery was performed by Exeter-based surgeons, with $68 \%$ of cases performed by teams led by surgeons with a specialist hip interest and $32 \%$ by general orthopaedic surgeons from other teams. The surgery was performed by a consultant in $70 \%$ of the cases and the remainder by more junior members of the team and fellows. We used a posterior approach in $78 \%$ of hips and the direct lateral approach in $22 \%$. Contemporary cementation techniques [35] were used in $88 \%$ of the cases, with earlier methods in the remainder. The contemporary technique included preparation of the acetabulum by reaming to remove the subchondral bone, creating multiple small drill holes, and pressurizing the cement using a balloon pressurizer. After femoral preparation and insertion of a cement restrictor, the cement was introduced to the femur in a retrograde fashion and then prolonged pressurization applied using a proximal Sorbothane ${ }^{\mathbb{R}}$ (Stryker Inc, Caen, France) seal, eliminating the method of finger packing. After implant insertion, the pressure was maintained using a proximal collar until cement polymerization.

The acetabular implant used was the Exeter ${ }^{\mathrm{TM}}$ concentric all-polyethylene cup (Stryker Inc, Caen, France) in $50 \%$ of hips, the Exeter ${ }^{\mathrm{TM}}$ metal-backed polyethylene cup (Stryker Inc) in $32 \%$, the Ogee ${ }^{\circledR}$ (DePuy, Leeds, UK) in $15 \%$, and the BiomEx ${ }^{\mathrm{TM}}$ (Biomet, Warsaw, Indiana) uncemented cup in 3\%. The femoral head in all cases was of modular construction from the same Orthinox ${ }^{\circledR}$ stainless steel as the stem. The head diameter was $26 \mathrm{~mm}$ in all but five hips, with $30 \mathrm{~mm}$ used in three cases and $22 \mathrm{~mm}$ used in two cases performed through the direct lateral approach. The Exeter ${ }^{\mathrm{TM}}$ Universal stem (Stryker Inc.) was used in every case.

The department followed patients yearly for 5 years, then 5-yearly review clinically and radiographically, with 
Fig. 1A-B (A) A preoperative radiograph demonstrates an example of the complex cases encountered. (B) A postoperative film of the same patient at 12 years shows well-fixed components.
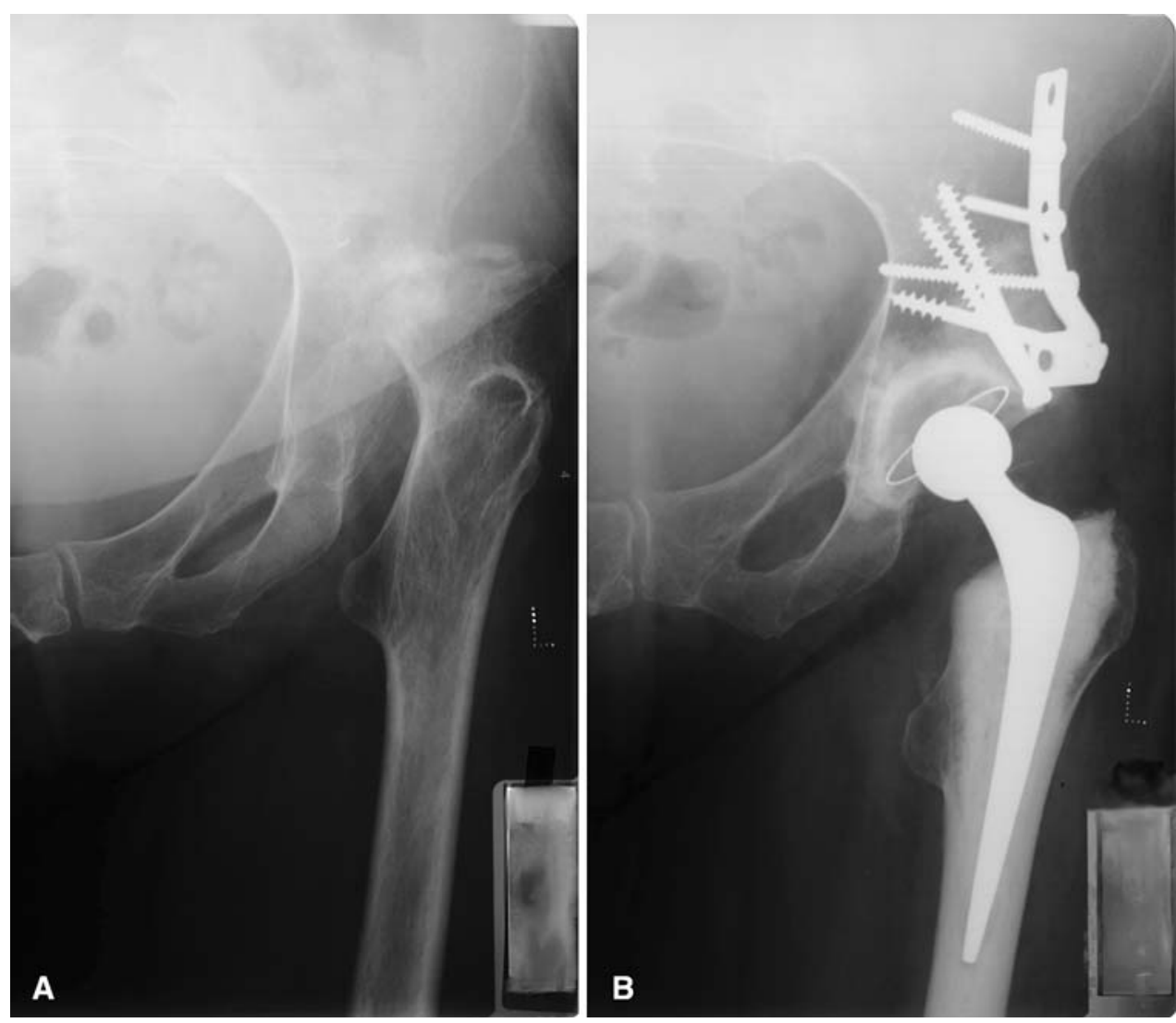

all information collected prospectively and entered into a database (Orthographics Inc, Salt Lake City, UT). To ensure current data, all patients were seen for review within 12 months of the study date, either as part of their standard followup or in extra clinics for the purpose of this study. Pre- and postoperative clinical scores included the Charnley modification of the Merle D'Aubigné-Postel score [11], Harris hip score [25], and Oxford hip score [15]. The University of California, Los Angeles (UCLA) activity score was assessed at last review [1, 52]. The general health status of the patients was assessed with the EuroQol EQ-5D score, which provides a single index value of health-related quality of life that is derived from a descriptive profile of five dimensions. The reference score derived from an age-matched random UK population sample is $79 \%$ [19]. The tool also provides a "thermometer" on a visual analog scale to generate a self-rated current health-related quality of life. The age-matched reference is $0.8[19,31]$.

All patients had anteroposterior and lateral radiographs preoperatively, postoperatively, and at each review. These radiographs were digitized and scaled using the known size of the femoral head implant. Despite the presence of a stem centralizer and consequent lack of cement at the immediate stem tip (there was cement below the tip to the level of the cement plug), we (SL, BS) assessed the quality of femoral cementation immediately postsurgery according to the system of Barrack et al. [3] because it predicts outcome [10]. On initial postoperative films, there were 40 Grade A femora, 51 Grade B, 27 Grade C, and two Grade D. In the 10 remaining stems, it was not possible to determine the cementation grade due to inadequate films or the presence of plates used for fixation of shortening and derotation osteotomies. A radiolucent line was defined as a sclerotic line adjacent to a linear radiolucency following the criteria described by Kobayashi et al. [33]. The position of lucent lines in the femur was described on the anteroposterior radiograph using zones of Gruen et al. [24] and on the lateral by Johnston et al. [27]. Osteolysis was defined as a progressive nonlinear lucency $(>2 \mathrm{~mm}$ ) resulting in endosteal erosion of cortical or cancellous bone [38]. Failure of the femoral construct was taken as progressive signs of radiolucency or migration of the cement mantle within the femur. Since stem subsidence is recognized as taper engagement and part of the normal behavior of the Exeter $^{\mathrm{TM}}$ stem, it was not used as a criterion for failure. We measured subsidence of the femoral stem using the method described by Fowler et al. [23] where the space between the shoulder of the prosthesis and the cement overlying it represents the amount of subsidence. Demarcation of acetabular components was expressed using zones described by DeLee and Charnley [16]. The classification of 
Hodgkinson et al. [26] was used to indicate acetabular loosening. A radiolucent line in all three zones or migration was believed to indicate loosening and failure of fixation. Linear cup wear was estimated with digital radiographs at normal magnification using the method described by Livermore et al. [36].

We constructed life tables to indicate survivorship using the technique described by Kaplan and Meier, [29] and 95\% confidence intervals (CIs) were calculated using the equation described by Peto et al. [41]. We included patients who died during the study. When sockets were revised, wellfixed stems were routinely removed to facilitate acetabular exposure and the original stem cement-bone interface was preserved. An in-cement replacement of stem was then carried out. Because the femoral cement-bone interface was not disturbed, these were not considered stem revisions in the survivorship analysis. We used the Student's t test to compare linear acetabular wear rates between failed and surviving sockets. We used the chi square test with Yates correction [51] to assess the relationship between the Barrack cement grade and femoral radiolucent lines. We compared stem subsidence in this cohort to a previously reported general group of patients [50].

\section{Results}

Our final review confirmed these were indeed high-activity patients: the mean UCLA activity score for all groups was 5.8 (standard deviation [SD], 1.9). Seventy-four percent of the patients maintained an active lifestyle indicated by a score of 5 and above. When patients in Charnley Group A were considered alone, the average activity score was 6.4 (SD, 1.9), with $83 \%$ of patients active at Level 5 or above and $69 \%$ at Level 7 or above, indicating regular sports (Table 3).

No stems were revised for aseptic loosening, giving a stem survival rate at 17 years of $100 \%$ (Fig. 2). The 10-year survival rate with reoperation for any reason as an end point was $94.4 \%$ (95\% CI, 89.3\%-97.9\%). At an average followup of 12.5 years, this was $92.6 \%(95 \%$ CI, 87.3-97.9) (Fig. 3). Of the 123 hips for review, 13 (10.6\%) had undergone further surgery by last review (Table 2). Using revision for aseptic loosening of the cup as an end point, the 10-year survival rate was $97.6 \%$ (95\% CI, 93.2\%-99.2\%). At 12.5 years, this was $95.9 \%$ (95\% CI, 89.4\%-98.5\%) (Fig. 4). Of the unrevised acetabular components, $78 \%$ had either no demarcation or minor changes in Zone 1, whereas $12.8 \%$ (14) of the cups had either demarcation in all zones or migration, thus fulfilling radiographic criteria for loosening (Fig. 5). Therefore, including the revisions, $23(18.7 \%)$ of the acetabular components were judged to have failed clinically or radiographically at the time of final review.

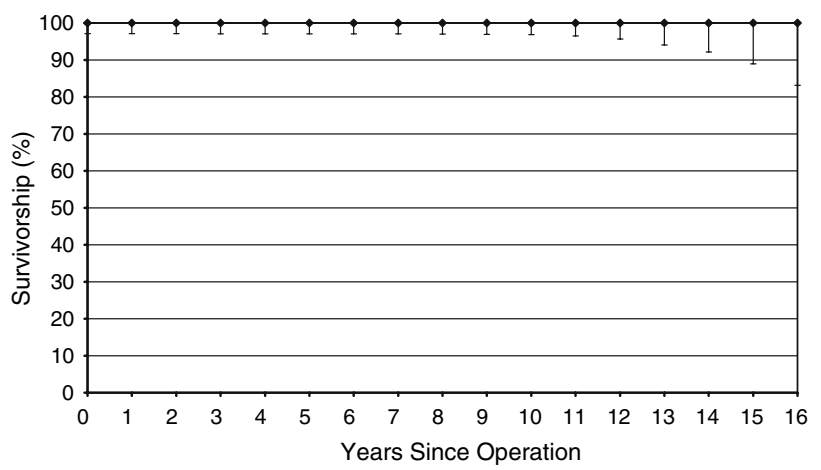

Fig. 2 A graph shows survivorship with any reoperation for aseptic stem loosening as an end point. Error bars $=95 \%$ confidence limits, obtained using the Peto equation [40].

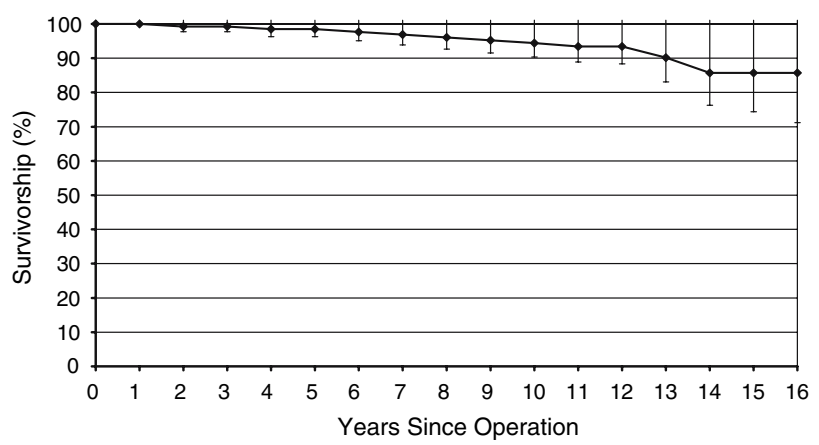

Fig. 3 A graph shows survivorship with any reoperation, including periprosthetic fractures, as an end point. Error bars $=95 \%$ confidence limits, obtained using the Peto equation [40].

The mean stem subsidence at review was $1.29 \mathrm{~mm}$ (range, $0-2.9 \mathrm{~mm} ; \mathrm{SD}, 0.45$ ). The only stem with no measurable subsidence was in a patient who was wheelchair-bound. Other activity levels were not associated with differing subsidence. There was no difference when comparing subsidence with cement grade.

The mean linear wear for all cups was $0.09 \mathrm{~mm}$ per year (range, $0-0.32 \mathrm{~mm}$ ). The wear rates in those cups revised for aseptic loosening were higher $(\mathrm{p}=0.002)$ at $0.21 \mathrm{~mm}$ per year. The linear wear rate for the surviving hips with a UCLA activity level less than 5 was $0.06 \mathrm{~mm}$ per year, whereas for those with scores from 5 to 10 it was higher $(\mathrm{p}=0.011)$ at $0.1 \mathrm{~mm}$ per year.

No femur had evidence of focal endosteal lysis. Twelve femurs showed evidence of radiolucent lines at final review. Of these, six had lucent lines at the calcar (Zone 7), five had lines in two proximal zones, and one case had radiolucent lines in four zones. This hip had a 30-mm-diameter head articulating with an Exeter ${ }^{\mathrm{TM}}$ metal-backed cup. The lines were nonprogressive and there was no evidence of subsidence of the cement within the bone. The patient was asymptomatic, scoring 6-6-6 on the Charnley modification of the D'Aubigné-Postel scale and with a Harris hip score of 
Table 2. Reoperation for any reason

\begin{tabular}{lllllll}
\hline Patient & Gender & $\begin{array}{l}\text { Age at } \\
\text { surgery (years) }\end{array}$ & Underlying diagnosis & $\begin{array}{l}\text { Years to } \\
\text { revision }\end{array}$ & Reason for reoperation & Comments \\
\hline 1 & Male & 32 & Avascular necrosis & 2.5 & Acetabular aseptic loosening & Socket only revised \\
2 & Female & 44 & Rheumatoid arthritis & 4.3 & Dislocation & Socket only revised \\
3 & Female & 48 & Osteoarthritis & 6.7 & Acetabular aseptic loosening & Socket only revised \\
4 & Female & 48 & Developmental dysplasia & 7.2 & Acetabular aseptic loosening & Socket only revised \\
5 & Male & 40 & Osteoarthritis & 8.5 & Periprosthetic fracture type C & Original components retained \\
6 & Female & 40 & Septic arthritis & 8.5 & Sepsis & Socket only revised; pain resolved \\
7 & Female & 18 & Perthes & 9.1 & Pain and acetabular lysis & Socket only revised \\
8 & Female & 44 & Osteoarthritis & 10.9 & Acetabular aseptic loosening & Socket only revised \\
9 & Female & 44 & Osteoarthritis & 11.1 & Acetabular aseptic loosening & Sock \\
10 & Male & 28 & Avascular necrosis & 13.3 & Acetabular aseptic loosening & Socket only revised \\
11 & Female & 43 & Developmental dysplasia & 13.7 & Acetabular aseptic loosening & Socket only revised \\
12 & Female & 36 & Slipped femoral epiphysis & 14.1 & Acetabular aseptic loosening & Socket only revised \\
13 & Female & 50 & Osteoarthritis & 14.3 & Acetabular aseptic loosening & Socket only revised \\
\hline
\end{tabular}

Table 3. University of California at Los Angeles Activity Score [1, 50]

\begin{tabular}{|c|c|c|c|}
\hline Score & Description & Percent for all groups & Percent for group A \\
\hline 1 & Very inactive, dependent on others; cannot leave home & 2.1 & 0 \\
\hline 2 & $\begin{array}{l}\text { Mostly inactive; restricted to minimal activities, } \\
\text { such as washing, dressing, toileting }\end{array}$ & 6.3 & 8.3 \\
\hline 3 & Limited housework, shopping; occasional walking & 3.1 & 0 \\
\hline 4 & Regular mild activities, such as housework, walking, seated occupation & 15.5 & 8.3 \\
\hline 5 & $\begin{array}{l}\text { Unlimited housework or shopping; occasional moderate activities, } \\
\text { such as swimming }\end{array}$ & 6.2 & 4.2 \\
\hline 6 & Regular moderate activity, such as swimming; light occupational work & 19.8 & 12.5 \\
\hline 7 & Regular sports, such as cycling, aerobics, gym, gardening once or twice a week & 29.2 & 37.5 \\
\hline 8 & $\begin{array}{l}\text { Regular very active events, such as bowling or golf, riding, aerobics; } \\
\text { gym or gardening three or more times a week; farming; } \\
\text { moderately heavy occupation }\end{array}$ & 16.7 & 25 \\
\hline 9 & $\begin{array}{l}\text { Occasional impact sports, such as running, jogging, tennis, cricket, } \\
\text { football, rugby, martial arts, skiing, ballet, hiking, mountaineering; } \\
\text { heavy occupation }\end{array}$ & 1.0 & 4.2 \\
\hline 10 & Regular impact sports as previously described & 0 & 0 \\
\hline
\end{tabular}

100. Of the 40 Barrack Grade A cemented femurs, three (7.5\%) had some evidence of lucent lines. Grade B cemented femurs (51) had an incidence of lines in two (3.9\%), whereas Grade C cementation (27) was associated with greater $(\mathrm{p}<0.01)$ incidence of lucent lines $(25.9 \%$, seven femora). The two Grade D cemented stems were in a patient who died 8 years postoperation but had no evidence of femoral osteolysis at that time.

The mean Harris hip score improved to a satisfactory level at final followup (from a preoperative level of $36 \pm 17$, range, $1-68$ to $81 \pm 19$, range, $18-100$ at final review). The mean Oxford score at final review was $21 \pm 8.5$ (range, 12-47). The overall mean Charnley modification of the Merle D'Aubigné-Postel score before surgery was 2.3 for pain, 2.3 for mobility, and 3.1 for range of movement. At final review, these scores were 5.2, 4.6, and 5.4, respectively (Table 4). The mean state of health on the EuroQol scale was $77 \%$ for all groups at final review. Those who were Charnley Group A scored 83\%. The mean weighted health status index for all groups was 0.67 , being 0.89 for Group A patients.

\section{Discussion}

The Exeter ${ }^{\mathrm{TM}}$ stem is associated with high survival rates at 12 years in all patients, but its performance in younger patients has not been established. We therefore asked 


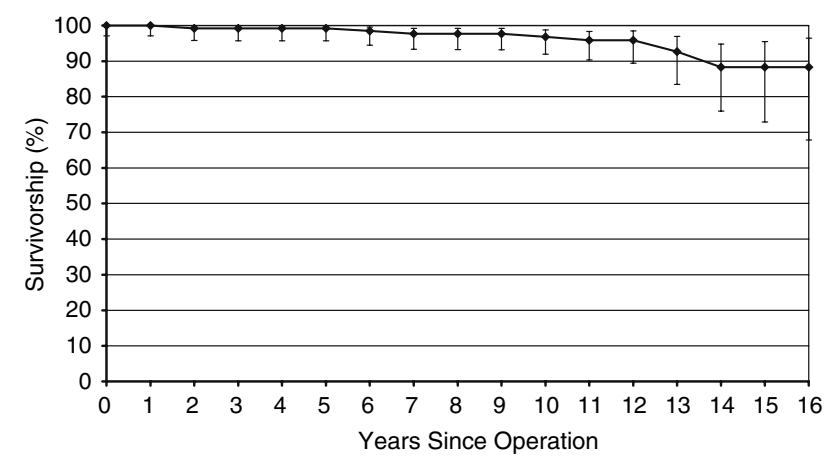

Fig. 4 A graph shows survivorship with reoperation for aseptic cup loosening as an end point. Error bars $=95 \%$ confidence limits, obtained using the Peto equation [40].

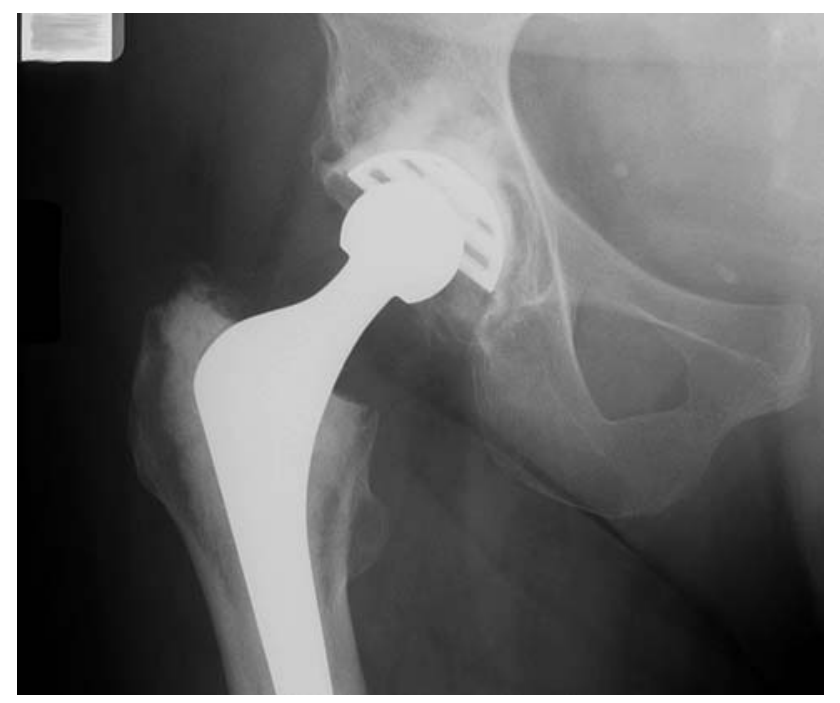

Fig. 5 A radiograph demonstrates linear socket wear and acetabular lysis behind a metal-backed cup at 15 years postsurgery.

Table 4. Charnley modification of Merle D'Aubigne-Postel Score [10]

\begin{tabular}{lllll}
\hline Group & Percent & Pain & Function & Range of movement \\
\hline Preoperative & & & & \\
A & 42.9 & 2.5 & 3.1 & 3.4 \\
B & 25.4 & 2.6 & 2.4 & 3.3 \\
C & 31.7 & 1.7 & 1.4 & 2.7 \\
All groups & & 2.3 & 2.3 & 3.1 \\
Final review & & & & \\
A & 26.9 & 5.2 & 5.5 & 5.5 \\
B & 45.2 & 5.4 & 5.4 & 5.5 \\
C & 27.9 & 4.9 & 3.0 & 5.3 \\
All groups & & 5.2 & 4.6 & 5.4 \\
\hline
\end{tabular}

whether the Exeter ${ }^{\mathrm{TM}}$ Universal hip performs as well in younger, more active patients as in patients of all ages by establishing if medium- to long-term survival rates were comparable. Having established whether these young patients were indeed active, we then sought to ascertain if increased wear rates were observed and associated with radiographic evidence of implant failure.

For logistic and financial reasons, we were unable to obtain complete data on all patients operated upon within the hospital, since some came from outside the region as did their surgeons and we had no control over their followup regimes. Thus, this series is of selected rather than consecutive cases in reference to all surgery performed within the hospital. However, when considering THAs performed by all surgeons based in Exeter, their fellows and juniors, none was omitted. We presume the range of surgeons involved is nonetheless representative of those operated by outside surgeons.

Survivorships of THAs in young patients reportedly have had generally inferior results in the past when compared with those of a general population with overall survival rates averaging $80 \%$ at 10 years in patients under 50 years compared to $92 \%$ at $15-20$ years in older patients [7, 13, 17, 20, 40, 47]. Younger patients are believed more active and so place more burden on their joint replacement in terms of higher cycle frequency and load [44-46]. These demands may lead to increased wear of the bearing surface $[32,43]$. It has been postulated, therefore, it is this "use" rather than chronologic age that determines wear and, indeed, patients with higher activity levels when measured on the UCLA scale had higher rates of acetabular polyethylene wear in this study. We subjectively assessed activity levels of this group of patients throughout their followup, but only at the last review did we introduce the UCLA activity scale into the evaluation. We recognize patients' activity levels after THA are highly variable and cumulative. Therefore, the patients with lower scores at last review may have been more active in the early years after THA and the patients with high scores may have been as active throughout the lifespan of their hip [18]. In both these scenarios, patient activity would tend to have been underrepresented by a single score. Despite this, $74 \%$ of our patients were still undertaking active lifestyles at an average of 12.5 years postsurgery.

The Exeter ${ }^{\mathrm{TM}}$ metal-backed cup was introduced in 1984 since it was believed to have theoretical advantages, but when the design became associated with excessive wear and early failure and the theory for metal backing was believed unsound by some [ $8,9,14]$, its use was abandoned in 1990. However, we found no association between metalbacked sockets and failure, possibly due to the low number of revisions encountered.

The sockets revised for aseptic loosening were associated with higher linear wear rates than the group as a whole. High rates of socket wear result in large volumes of particulate polyethylene debris, which stimulates osteolysis 
at cement-bone interfaces. In studies of other cemented stems, this is also associated with high rates of femoral component failure of $13 \%$ at 25 years to $18 \%$ at 12.8 years $[7,12,28]$. However, in our patients the femoral cementbone interface in such cases seemed durable. It has been postulated, in the case of the Exeter ${ }^{\mathrm{TM}}$ stem, that this is the result of the protective effects the compressive stem biomechanics have on this boundary [49].

Although the Exeter ${ }^{\mathrm{TM}}$ stem has been said to have a forgiving biomechanical behavior [50], it is important to emphasize the method of cementation of the femur must be meticulous because, as in previous studies [3, 48], results demonstrating poor cementation were associated with the appearance of radiolucent lines in the medium to long term.

The Exeter ${ }^{\mathrm{TM}}$ Universal series of 325 hips reported by Williams et al. [50] in 2002 demonstrated a survivorship rate with reoperation for any cause of $91.74 \%$ at 12 years. The study presented here of young patients has a comparable survivorship rate of $92.6 \%$ at 12.5 years. The revision rate for aseptic cup loosening was also similar with survivorship rates of $96.86 \%$ and $95.9 \%$, respectively. The earlier paper, however, was made up of $94 \%$ metal-backed cups compared with $32 \%$, with a lack of association between metal backing and failure in this group. This suggests factors other than the presence of a metal-backed socket contributed to failure, such as increased activity and consequent polyethylene wear rates. Neither series encountered revision for aseptic stem loosening. Our data were similar to those in the other study in other respects. The stem subsidence rate found in our series $(1.29 \mathrm{~mm})$ was comparable to that found by Williams et al. [50] $(1.32 \mathrm{~mm})$. The relationship of increased subsidence with poorer cementation grade was not, however, observed in this cohort. The incidence of radiolucent lines was $8.9 \%$ in the 2002 study and $9.8 \%$ in our study, with the majority appearing in the proximal zones. The correlation between Barrack $\mathrm{C}$ cementation grade and the presence of radiolucent lines is a new finding in this series. When compared with other reviews of cemented THAs in young people, the Exeter $^{\mathrm{TM}}$ hip performs extremely well. Callaghan et al. [7] reporting on Charnley THAs in patients less than 50 years of age, demonstrated a 12-year survival rate for reoperation of approximately $85 \%$; other studies reported similar results. There was a general finding of better performance of the stems than the sockets [5, 12, 28, 30]. McAuley et al. [37] reported a survivorship rate for reoperation of $88.8 \%$ at 10 years in young patients compared with $94.4 \%$ at the same point in time in our study. The Exeter ${ }^{\mathrm{TM}}$ also compares well with other studies of uncemented THAs in young patients with a similar length of followup [21, 34, 39]. These studies, all with patients younger than 50 years old, had survival rates of between $70.7 \%$ and $92.5 \%$ for all causes with a maximum followup of 14 years. Once again, socket failures outnumbered stems, but all encountered stem revisions for aseptic loosening, with femoral failure rates of between $1.1 \%$ and $15.1 \%$.

Our data suggest the Exeter ${ }^{\mathrm{TM}}$ Universal stem provides high survivorship in the medium- to long-term, even when used in young, high-demand patients, and can be implanted confidently in such cases. Despite poorer performance of the various sockets, the survival rate with an end point of reoperation for any reason was $94.4 \%$ at 10 years and $92.6 \%$ at a mean followup of 12.5 years. It is expected, when coupled with acetabular components less prone to wear, it is likely to exhibit further improvements in overall performance.

Acknowledgments We thank members of the Exeter Hip Unit, whose efforts ensured this series of patients have received meticulous followup: Ros Sculpher, Sandy Wraight, and Zoë Tippett.

\section{References}

1. Amstutz HC, Thomas BJ, Jinnah R, Kim W, Grogan T, Yale C. Treatment of primary osteoarthritis of the hip: a comparison of total joint and surface replacement arthroplasty. $J$ Bone Joint Surg Am. 1984;66:228-241.

2. Ballard WT, Callaghan JJ, Sullivan PM, Johnston RC. The results of improved cementing techniques for total hip arthroplasty in patients less than fifty years old: a ten-year follow-up study. J Bone Joint Surg Am. 1994;76:959-964.

3. Barrack RL, Mulroy RD Jr, Harris WH. Improved cementing techniques and femoral component loosening in young patients with hip arthroplasties: a 12-year radiographic review. J Bone Joint Surg Br. 1992;74:385-389.

4. Beckenbaugh RD, Ilstrup DM. Total hip arthroplasty: a review of 333 cases with long follow up. J Bone Joint Surg Am. 1978;60:306-13.

5. Bizot P, Hannouche D, Nizard R, Witvoet J, Sedel L. Hybrid alumina total hip arthroplasty using a press-fit metal-backed socket in patients younger than 55 years: a six- to 11-year evaluation. J Bone Joint Surg Br. 2004;86:190.

6. Boeree NR, Bannister GC. Cemented total hip arthroplasty in patients younger than 50 years of age: ten- to 18-year results. Clin Orthop Relat Res. 1993;287:153-159.

7. Callaghan JJ, Forest EE, Olejniczak JP, Goetz DD, Johnston RC. Charnley total hip arthroplasty in patients less than fifty years old: a twenty to twenty-five-year follow-up note. J Bone Joint Surg Am. 1998;80:704-714.

8. Carter DR, Vasu R, Harris WH. Stress distributions in the acetabular region. II. Effects of cement thickness and metal backing of the total hip acetabular component. J Biomech. 1982;15:165170.

9. Cates HE, Faris PM, Keating EM, Ritter MA. Polyethylene wear in cemented metal-backed acetabular cups. J Bone Joint Surg Br. 1993;75:249-253.

10. Chambers IR, Fender D, McCaskie AW, Reeves BC, Gregg PJ. Radiological features predictive of aseptic loosening in cemented Charnley femoral stems. J Bone Joint Surg Br. 2001;83:838-842.

11. Charnley J. Numerical grading of clinical results. In: Low Friction Arthroplasty of the Hip: Theory, Practice. New York, NY: Springer; 1979:20-24.

12. Chmell MJ, Scott RD, Thomas WH, Sledge CB. Total hip arthroplasty with cement for juvenile rheumatoid arthritis: results 
at a minimum of ten years in patients less than thirty years old. J Bone Joint Surg Am. 1997;79:44-52.

13. Collis DK. Long-term (twelve to eighteen-year) follow-up of cemented total hip replacements in patients who were less than fifty years old: a follow-up note. J Bone Joint Surg Am. 1991;73:593-597.

14. Dalstra M, Huiskes R. The influence of metal-backing in cemented cups. Orthop Trans. 1991;15:459.

15. Dawson J, Fitzpatrick R, Carr A, Murray DW. Questionnaire on the perception of patients about total hip replacement. $J$ Bone Joint Surg Br. 1996;78:185-190.

16. DeLee JG, Charnley J. Radiological demarcation of cemented sockets in total hip replacement. Clin Orthop Relat Res. 1976;121:20-32.

17. Devitt A, O'Sullivan T, Quinlan W. 16- to 25-year follow-up study of cemented arthroplasty of the hip in patients aged 50 years or younger. J Arthroplasty. 1997;12:479-489.

18. Dolan P, Gudex C, Kind P, Williams A. A Social Tariff for the EuroQol: Results From a UK General Population Survey. Discussion paper 138. York, UK: University of York, Centre for Health Economics; 1995.

19. Dorey FJ. Survivorship analysis of surgical treatment of the hip in young patients. Clin Orthop Relat Res. 2004;418:23-28.

20. Dorr LD, Kane TJ 3rd, Conaty JP. Long-term results of cemented total hip arthroplasty in patients 45 years old or younger: a 16-year follow-up study. J Arthroplasty. 1994;9:453-456.

21. Duffy GP, Berry DJ, Rowland C, Cabanela ME. Primary uncemented total hip arthroplasty in patients $<40$ years old: 10 - to 14year results using first-generation proximally porous-coated implants. J Arthroplasty. 2001;16(Suppl 1):140-144.

22. EN58J. British Standard 3531-2. 1952.

23. Fowler JL, Gie GA, Lee AJ, Ling RS. Experience with the Exeter total hip replacement since 1970. Orthop Clin North Am. 1988;19:477-489.

24. Gruen TA, McNeice GM, Amstutz HC. "Modes of failure" of cemented stem-type femoral components: a radiographic analysis of loosening. Clin Orthop Relat Res. 1979;141:17-27.

25. Harris WH. Traumatic arthritis of the hip after dislocation and acetabular fracture: treatment by mold arthroplasty. J Bone Joint Surg Am. 1969:51:737-755.

26. Hodgkinson JP, Shelley P, Wroblewski BM. The correlation between the roentgenographic appearance and operative findings at the bone-cement junction of the socket in Charnley low friction arthroplasties. Clin Orthop Relat Res. 1988;228:105-109.

27. Johnston RC, Fitzgerald RH Jr, Harris WH, Poss R, Muller ME, Sledge CB. Clinical and radiographic evaluation of total hip replacement: a standard system of terminology for reporting results. J Bone Joint Surg Am. 1990;72:161-168.

28. Joshi AB, Porter ML, Trail IA, Hunt LP, Murphy JC, Hardinge K. Long term results of Charnley low-friction arthroplasty in young patients. J Bone Joint Surg Br. 1993;75:616-623.

29. Kaplan EL, Meier P. Nonparametric estimation from incomplete observations. J Am Stat Assoc. 1958;53:457-481.

30. Kerboull L, Hamadouche M, Courpied JP, Kerboull M. Long term results of Charnley-Kerboull hip arthroplasty in patients younger than 50. Clin Orthop Relat Res. 2004;418:112-118.

31. Kind P, Dolan P, Gudex C, Williams A. Variations in population health status: results from a United Kingdom national questionnaire survey. BMJ. 1998;316:736-741.

32. Kobayashi S, Eftekhar N, Terayama K, Joshi RP. Comparative study of total hip arthroplasty between younger and older patients. Clin Orthop Relat Res. 1997;339:140-151.

33. Kobayashi S, Takaoka K, Saito N, Hisa K. Factors affecting aseptic failure of fixation after primary Charnley total hip arthroplasty: multivariate survival analysis. J Bone Joint Surg Am. 1997;79:1618-1627.

34. Kronick JL, Barba ML, Paprosky WG. Extensively coated femoral components in young patients. Clin Orthop Relat Res. 1997;344:263-274.

35. Ling R. Cementing technique in the femur. Tech Orthop. 1991;6:34-39.

36. Livermore J, Ilstrup D, Morrey B. Effect of femoral head size on wear of the polyethylene acetabular component. J Bone Joint Surg Am. 1990;72:518-528.

37. McAuley JP, Edward S, Szuszczewicz ES, Young A, Engh CA Sr. Total hip arthroplasty in patients 50 years and younger. Clin Orthop Relat Res. 2004;418:119-125.

38. Mulroy WF, Estok DM, Harris WH. Total hip arthroplasty with use of so-called second generation cementing techniques: a fifteen-year-average follow-up study. J Bone Joint Surg Am. 1995;77:1845-1852.

39. Nercessian OA, Wu WH, Sarkissian H. Clinical and radiographic results of cementless AML total hip arthroplasty in young patients. J Arthroplasty. 2001;16:312-316.

40. Older J. Charnley low-friction arthroplasty: a worldwide retrospective review at 15 to 20 years. J Arthroplasty. 2002;17:675680.

41. Peto R, Pike MC, Armitage P, Breslow NE, Cox DR, Howard SV, Mantel N, McPherson K, Peto J, Smith PG. Design and analysis of randomised clinical trials requiring prolonged observation of each patient. Br J Cancer. 1977;35:1-40.

42. Rex 734. ISO 5832-9. 1992.

43. Schmalzried TP, Huk OL. Patient factors and wear in total hip arthroplasty. Clin Orthop Relat Res. 2004;418:94-97.

44. Schmalzried TP, Shepherd EF, Dorey FJ, Jackson WO, de la Rosa M, Fa'vae F, McKellop HA, McClung CD, Martell J, Moreland JR, Amstutz HC. The John Charnley Award. Wear is a function of use, not time. Clin Orthop Relat Res. 2000;381: $36-46$.

45. Schmalzried TP, Szuszczewicz ES, Northfield MR, Akizuki KH, Frankel RE, Belcher G, Amstutz HC. Quantitative assessment of walking activity after total hip or knee replacement. J Bone Joint Surg Am. 1998;80:54-59.

46. Silva M, Shepherd EF, Jackson WO, Dorey FJ, Schmalzried TR. Average patient walking activity approaches 2 million cycles per year: pedometers under-record walking activity. J Arthroplasty. 2002;17:693-697.

47. Sullivan PM, MacKenzie JR, Callaghan JJ, Johnston RC. Total hip arthroplasty with cement in patients who are less than fifty years old: a sixteen to twenty-two-year follow-up study. $J$ Bone Joint Surg Am. 1994;76:863-869.

48. Sutherland CJ, Wilde AH, Borden LS, Marks KE. A 10-year follow up of one hundred consecutive Müller curved-stem total hip-replacement arthroplasties. J Bone Joint Surg Am. 1982;64: 970-82.

49. Wheeler JP, Miles AW, Clift S. The influence of stem-cement interface in total hip replacement-a comparison of experimental and finite element approaches. Proc Inst Mech Eng $H$. 1997;211:181-186.

50. Williams HD, Browne G, Gie GA, Ling RS, Timperley AJ, Wendover NA. The Exeter cemented femoral component at 8-12 years-a clinical, radiological and survivorship study of the first 325 cases. J Bone Joint Surg Br. 2002;84:324-334.

51. Yates F. Contingency table involving small numbers and the $\chi^{2}$ test. J R Stat Soc. 1934;1:217-235.

52. Zahiri C, Schmalzried TP, Szuszczewicz ES, Amstutz HC. Assessing activity in joint replacement patients. J Arthroplasty. 1998;12:890-895. 